\title{
Soft Substrate Induces Endothelial Cell Inflammation and Disrupts Endothelium Integrity
}

\author{
Yiling Tan*, Xiuli Mao, Huanli Wang \\ School of Biomedical Engineering, Shanghai Jiao Tong University, Shanghai, China \\ Email: ${ }^{\star} 18862185264 @ 163 . c o m$
}

How to cite this paper: Tan, Y.L., Mao, X.L. and Wang, H.L. (2021) Soft Substrate Induces Endothelial Cell Inflammation and Disrupts Endothelium Integrity. Journal of Biosciences and Medicines, 9, 92-102. https://doi.org/10.4236/jbm.2021.92009

Received: January 18, 2021

Accepted: February 21, 2021

Published: February 24, 2021

Copyright $\odot 2021$ by author(s) and Scientific Research Publishing Inc. This work is licensed under the Creative Commons Attribution International License (CC BY 4.0).

http://creativecommons.org/licenses/by/4.0/

\section{(c) (i) Open Access}

\begin{abstract}
Atherosclerosis (AS) is the main cause of death and disability all over the world. A lot of efforts have been devoted to treat AS, among which tissue engineering blood vessel materials, including artificial blood vessels, stents and vascular patches, have brought hope to ameliorate the symptoms in AS patients. However, there remains a large percentage of implantation failure due to the incompatibility of the material with the body. AS is a multi-factor related disease, and chronic inflammation is a major event that involves with its pathogenesis and development. Since previous studies suggested that the stiffness of the blood vessel might affect the inflammatory conditions, in this paper, we investigate the mechanism of how substrate stiffness could affect the inflammation response of the endothelial cells (ECs). Polyacrylamide (PA) based hydrogels at different concentrations were used as the culture substrate for ECs. The mRNA expression level of VCAM-1 and ICAM-1 was determined by qRT-PCR. EC chemotactic effect was evaluated by the number of THP- 1 adhered to EC monolayer. The protein levels of $\mathrm{I} \kappa \mathrm{B} \alpha$ and NF- $\kappa \mathrm{B}$ were determined by western blotting analysis. The expression and localization of the major adherens junctions (AJs) proteins, VE-cadherin and $\beta$-catenin, were evaluated by western blotting and immunofluorescence staining. Our results showed that ECs cultured on soft substrate $(1 \mathrm{kPa})$ demonstrated more chemotactic effect and the amount of the monocytes adhered to them was higher than that on harder substrate $(20 \mathrm{kPa}, \mathrm{p}<0.05)$. Moreover, NF- $\kappa \mathrm{B}$ signaling pathway in ECs on $1 \mathrm{kPa}$ substrate was more activated compared to those on $20 \mathrm{kPa}$ substrate, with the $\mathrm{I} \kappa \mathrm{B} \alpha$ protein expression level in the cytoplasm decreasing and NF- $\kappa \mathrm{B}$ translocating more into the nuclear. In addition, the AJs of the endothelial monolayer changed with the substrate stiffness. Compared with ECs on normal substrate $(20 \mathrm{kPa})$, the protein expression level of $\beta$-catenin decreased $(\mathrm{p}<0.05)$, and immunofluorescence staining of
\end{abstract}


VE-cadherin and $\beta$-catenin showed the AJs between the ECs on soft substrate $(1 \mathrm{kPa})$ were punctuated. Taken together, our results suggested the stiffness of the substrate was important in regulating inflammation of the ECs and the integrity of the cell-cell junction. Therefore, the stiffness of the tissue engineering blood vessel material should be considered as an important criterium to avoid EC inflammation.

\section{Keywords}

Substrate Stiffness, Endothelial Cells, Inflammation, Adherens Junction

\section{Introduction}

Cardiovascular diseases are life-threatening, which are mainly caused by atherosclerosis (AS). Dyslipidemia, hypertension, diabetes and genetic factors are all closely related with the etiology of AS [1]. AS can result in the building up of the AS plaques and narrowing of the arteries, both significantly reducing the blood supply to vital organs such as the heart, brain and intestines. The formation of the plaque is essentially the result of unregulated inflammation. Many different kinds of pre-inflammatory factors, such as TNF- $\alpha$ and IL- $1 \beta$ have been proved to take part in the inflammation progress of AS [2]. AS preferentially takes place in the vascular areas exposed to disturbed flow and disturbed flow has been widely proved as a biophysical factor to induce vascular endothelial cells (ECs) dysfunctions [3] [4]. With the initiation of AS plaque formation, the stiffness of the vessel decreases as lipoprotein accumulating under the endothelium [5]. Vascular cells, including ECs and smooth muscle cells, display different functional changes to adapt to such biophysical microenvironmental change [6] [7].

Endothelium is the most important structure of the blood vessel to maintain its integrity and physiological function, as well as mediate the communication and interaction of the exterior environment in the circulation and the interior tissue. In healthy vessels, the intact endothelium can prevent the circulating cells in the blood from entering the intima. However, aging or pathological conditions will lead to accumulation of various kinds of lipoproteins to create AS prone sites in the vessel [8]. At this early stage of AS, the accumulation of the lipoproteins under the endothelium leads to significantly decreased vessel stiffness. Such change in stiffness may be sensed by ECs and ECs are therefore activated and secret cytokines to attract the circulating monocytes to adhere to the lesion sites and migrate into the intima [9] [10]. After the translocation, monocytes differentiate to macrophages and participate in the subsequent AS progress.

It has been reported that both transcellular and paracellular ways could be found in monocytes extravasation, but transcellular transport facilitated by impaired cell-cell junctions still plays dominant roles. ECs connect to each other with three main junctions, gap junctions, tight junctions and adherens junctions 
(AJs) [11], among which the AJs regulate the permeability of the endothelium and act as a gatekeeper of the endothelial barrier. VE-cadherin is the main component of AJs and its steady state determines the AJs integrity. As a transmembrane adhesion protein, the extracellular region of VE-cadherin mediates the direct connection between cells and the intracellular region is coupled to the cytoskeleton by proteins like $\beta$-catenin, which helps stabilize the AJs complex [12]. Besides, the ECs inflammation influences AJs integrity, with many proinflammatory factors reported to uncouple VE-cadherin from the actin cytoskeleton [13] [14] [15].

According to previous research, the stiffness of the healthy aorta is around 20 $\mathrm{kPa}$ and that of the AS site is around $1 \mathrm{kPa}$ [6] [16]. Therefore, we fabricated polyacrylamide hydrogel with stiffness of $1 \mathrm{kPa}$ and $20 \mathrm{kPa}$ to mimic the injured blood vessel at the early stage of AS and healthy blood vessel to study how the softening of substrate stiffness affects the ECs inflammatory response and vascular integrity. This helps to further understand the mechanism of AS in the early stage and may provide some hints for the treatment of the disease.

\section{Materials and Methods}

\subsection{Cell Cultural}

Human umbilical vein endothelial cells (HUVECs) (Sciencell, USA) were cultured in Endothelial Cell Medium supplemented with 5\% FBS, 1\% EC growth factor and $1 \%$ penicillin/streptomycin (Sciencell, USA). Confluent cells with the passage less than 10 were used in experiments. THP-1 monocyte cells purchased from American Type Culture Collection were cultured in RPMI Medium Modified (HyClone, USA) supplemented with 10\% FBS (HyClone, USA) and 1\% penicillin/streptomycin (Sciencell, USA). All the cells were cultured in the $37^{\circ} \mathrm{C}$ incubator with $5 \% \mathrm{CO}_{2}$.

\subsection{Manufacturing of Polyacrylamide Hydrogels}

Polyacrylamide (PA) hydrogels were manufactured as described before [17] [18]. Briefly, the solutions of $1 \mathrm{kPa}$ PA gel was prepared by mixing $6 \%$ acrylamide (Sangon Biotech, China) and 0.05\% bis-acrylamide (Sangon Biotech, China), and the solutions of $20 \mathrm{kPa}$ PA gel was prepared by mixing $8 \%$ acrylamide and $0.264 \%$ bis-acrylamide. Polymerization was initiated with $0.1 \%$ tetramethylethylenediamine (TEMED, Klamar, China) and $0.01 \%$ ammonium persulfate solution. To make a substrate suitable for each well in a 6-well culture dish, $200 \mu$ of the final solution was dropped onto a glass slide pre-treated with gel slick (Lon$\mathrm{za}$, Switzerland), and a silanized coverslip $30 \mathrm{~mm}$ in diameter was placed on the top of the solution. The PA gel yielded was $30 \mathrm{~mm}$ in diameter and $200 \mu \mathrm{m}$ in thickness. After 10 min of polymerization, the glass slide was removed and the coverslip with the gel attached was treated with sulfo-SANPAH (ProteoChem, USA) under UV for $15 \mathrm{~min}$ and coated with $0.1 \mathrm{mg} / \mathrm{ml}$ rat collagen I (Corning, USA). Before cell seeding, the gels were sterilized by UV for 30 minutes and 75\% ethanol. 


\subsection{Cell Seeding}

The gels were placed in the cell culture plate with the gel side up and the coverslip side down. The ECs were seeded on the gels with the concentration of 1.5 $2.0 \times 10^{5} / \mathrm{ml}$. Cells were cultured to reach $100 \%$ confluent before being used for any experiment.

\subsection{Western Blot Analysis}

Cells were washed with PBS and lysed by RIPA supplemented with protease inhibitors (Beyotime, China) according to manufacturer's instruction. The lysates were centrifuged at $12,000 \mathrm{rpm}$ for $5 \mathrm{~min}$ at $4^{\circ} \mathrm{C}$ to extract the whole protein in cells. Protein in the nuclear was extracted with the Nuclear and Cytoplasmic Protein Extraction Kit (Beyotime, China). The protein extracted was subjected to SDS-PAGE and then transferred to nitrocellulose membranes (Millipore, USA). After blocking with 10\% BSA (Sangon Biotech, China) at room temperature for 2 hours, the membranes were incubated overnight at $4^{\circ} \mathrm{C}$ with the following primary antibodies respectively: anti- $\mathrm{k} \mathrm{B} \alpha$, anti-NF- $\kappa \mathrm{B}$, anti- $\alpha$-tubulin, anti-VE-cadherin, anti- $\beta$-catenin, all from Cell Signaling Technology, USA and $\beta$-actin from Abcam, US. The membranes were then washed and incubated with HRP-conjugated secondary antibodies for 2 hours at room temperature. Finally, ECL substrate (Millipore, USA) was added to the membrane to visualize the protein on the membrane. The protein expression in each sample was normalized by the expression of $\alpha$-tubulin or $\beta$-actin in respective samples. Quantitation of the protein expression was processed with ImageJ.

\subsection{Quantitative Real-Time PCR Analysis}

RNA simple total RNA extraction kit was used for the extraction of total RNA. Fastking RT kit with gDNase was used for the reverse transcription and Talent qPCR PreMix (SYBR Green) kit was used in quantification real-time RCR (Tiangen Biotech, China). Relative gene expression levels were normalized to GAPDH. All the primers were purchased from BioTNT, China.

\subsection{Monocyte Adhesion Assay}

After centrifugation for $3 \mathrm{~min}$ at $800 \mathrm{rpm}$, THP-1 cells were resuspended with FBS-free RPMI medium to the concentration of $10^{6} / \mathrm{ml}$ and incubated with $2 \mu \mathrm{M}$ Calcein $\mathrm{AM}$ at $37^{\circ} \mathrm{C}$ for $15 \mathrm{~min}$. Then the labeled THP-1 was washed with DPBS and resuspended with Endothelial Cell Medium, added to the endothelial monolayer and incubated for 2 hours at $37^{\circ} \mathrm{C}$ in the incubator. Non-adherent cells were washed away with DPBS and the adherent cells were imaged with a fluorescence microscope (Leica, Germany). Three fields were of each sample were randomly selected to count adherent THP-1 cells.

\subsection{Immunofluorescence}

The EC monolayer on the PA gels was fixed with 4\% paraformaldehyde for 10 
min and washed with PBS for 3 times. Then, the cells were permeabilized with $0.1 \%$ tritonX-100, blocked with $5 \%$ BSA and incubated with primary antibody against VE-cadherin or $\beta$-catenin (Cell Signaling Technology, USA, 1:500) overnight at $4^{\circ} \mathrm{C}$. After washed with PBS, cells were incubated with fluorescent-labeled secondary antibody (Cell Signaling Technology, USA, 1:500) for 2 hours at room temperature in the dark. And DAPI (Beyotime, China, 1:5000) was used to counterstain the nucleus. Finally, the sample was covered with antifade mounting medium (Sigma, USA) and observed under a confocal microscope (Leica, TCS SP5 II, Germany).

\subsection{Statistical Analysis}

Unless otherwise indicated, the results were shown as the mean of at least three independent experiments, and error bars represent the standard error of the mean (SEM). Pairwise comparisons were made using a Student t-test. In all cases, $\mathrm{p}$ values less than 0.05 were considered statistically significant. Graph Pad Prism 6.0 software was used for all statistical evaluations.

\section{Results}

\subsection{Soft Substrate Induced ECs to Adhere More Monocytes}

As the initiation of the inflammatory responses in AS, monocyte will attach to the surface of the ECs. Activated ECs over-express VCAM1 and ICAM1 to facilitate monocyte adhesion [19]. We found that the mRNA expression levels of VCAM1 and ICAM1 were higher in the ECs monolayer seeded on the soft substrate (20 $\mathrm{kPa}$ as the control) (Figure 1(A)). About 2-fold more THP-1 monocytes adhered to the endothelial monolayer cultured on $1 \mathrm{kPa}$ substrate, compared with those on $20 \mathrm{kPa}$ substrate (Figure 1(B)). These results suggested that $1 \mathrm{kPa}$ substrate could activate ECs and induce inflammatory response.

\subsection{ECs Cultured on Soft Substrate Showed More NF- $\kappa$ B Activity}

$\mathrm{NF}-\kappa \mathrm{B}$ is an inflammation related transcription factor and its activation has been reported to corelate with the expression levels of VCAM1 and ICAM1 [20] [21] [22]. In NF- $\kappa \mathrm{B}$ signaling pathway, $\mathrm{I} \kappa \mathrm{B} \alpha$ is an important regulator that serves as an inhibitor of NF- $\kappa$ B. Before activation, $\mathrm{I} \kappa \mathrm{B} \alpha$ binds to NF- $\kappa \mathrm{B}$ and detains it in the cytoplasm [23]. The protein level of $\mathrm{I} \kappa \mathrm{B} \alpha$ was lower in the ECs cultured on 1 $\mathrm{kPa}$ substrate than those on $20 \mathrm{kPa}$ substrate (Figure 2(A)). Besides, the ECs on $1 \mathrm{kPa}$ substrate showed more NF- $\kappa \mathrm{B}$ protein expression in the nucleus (Figure $2(B))$. These results suggested that the NF- $\kappa \mathrm{B}$ signaling pathway was more activated in ECs cultured on soft substrate $(1 \mathrm{kPa})$. Collectively, soft substrate could induce more monocytes to adhere to ECs by activating NF- $\kappa \mathrm{B}$ nuclear translocation.

\subsection{Soft Substrate Induced Impaired AJs between ECs}

AJs between ECs play a very important role in regulating the permeability to circulating monocytes and solutes [24] [25]. Immunostaining showed that 
A
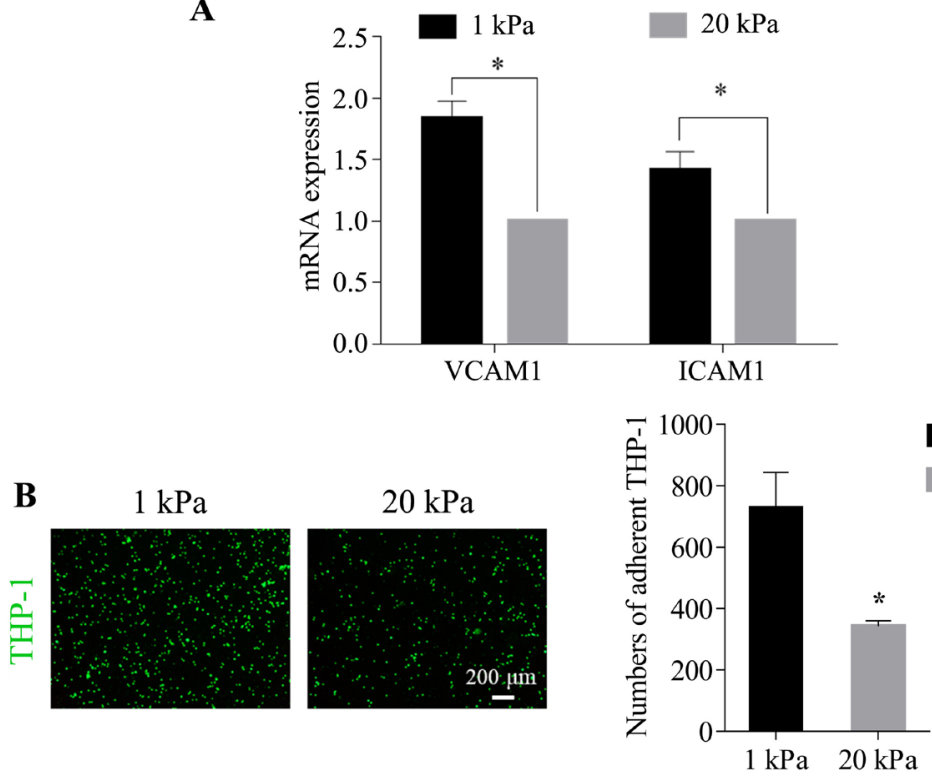

$1 \mathrm{kPa}$

$20 \mathrm{kPa}$

Figure 1. Soft substrate induced ECs to adhere more monocytes. (A) The mRNA expression levels of VCAM1 and ICAM1 in ECs seeded on $1 \mathrm{kPa}$ and $20 \mathrm{kPa}$ substrate was analyzed by RT-PCR. GAPDH was used as the internal control. $n=3,{ }^{*} \mathrm{p}<0.05$; (B) Calcein AM-labeled THP-1 cells were incubated with ECs monolayer cultured on $1 \mathrm{kPa}$ and 20 $\mathrm{kPa}$ substrate at $37^{\circ} \mathrm{C}$ for 2 hours. The number of THP-1 adhering to ECs monolayer was counted and compared. ${ }^{\star} \mathrm{p}<0.05$.

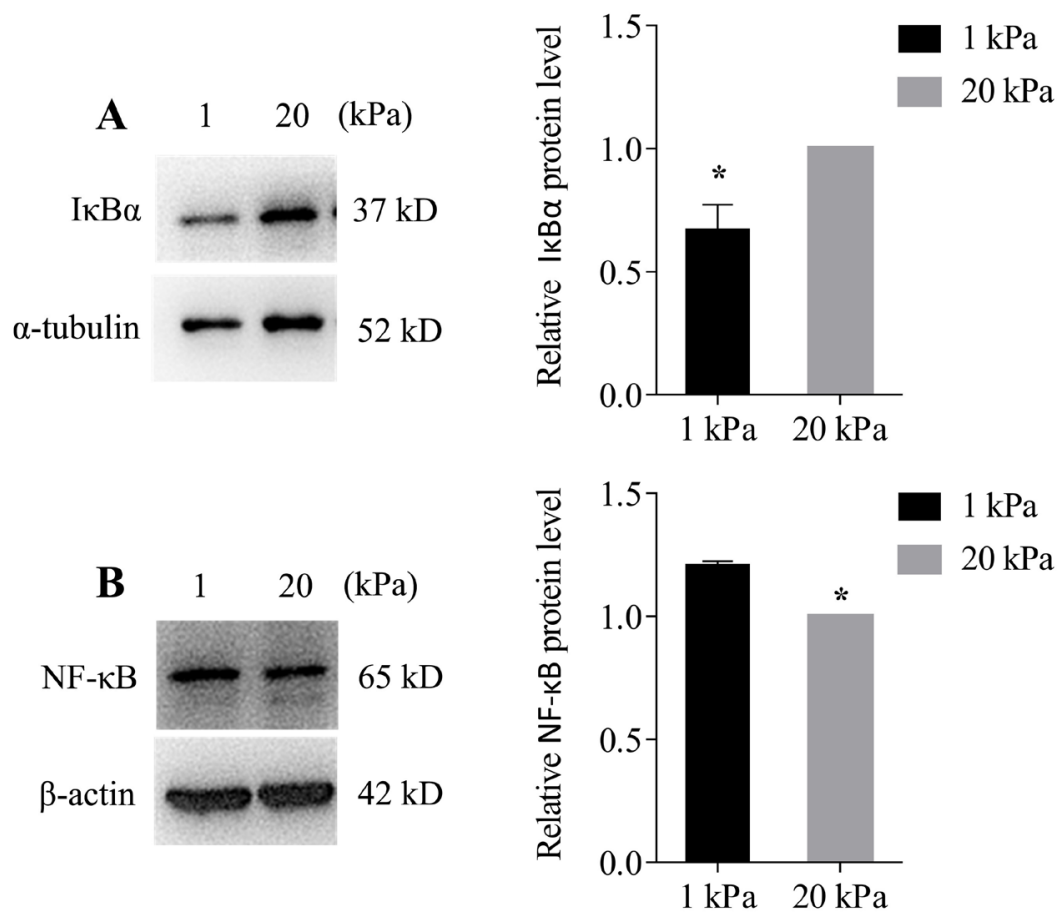

Figure 2. ECs cultured on soft substrate showed more NF- $\kappa$ B activity. ECs were cultured to reach a monolayer on PA gels, (A) The protein expression level of $\mathrm{I} \kappa \mathrm{B} \alpha$ was analyzed by immunoblotting. $\mathrm{n}=3,{ }^{\star} \mathrm{p}<0.05$; (B) NF- $\kappa$ B protein expression level in nuclear was analyzed by immunoblotting. $\mathrm{n}=3,{ }^{\star} \mathrm{p}<0.05$. (In all the experiments, $20 \mathrm{kPa}$ was used as the control.) 
VE-cadherin and $\beta$-catenin displayed punctuated distribution between ECs in the endothelial monolayer on $1 \mathrm{kPa}$ substrate (Figure 3(A), Figure 3(C)). Although,
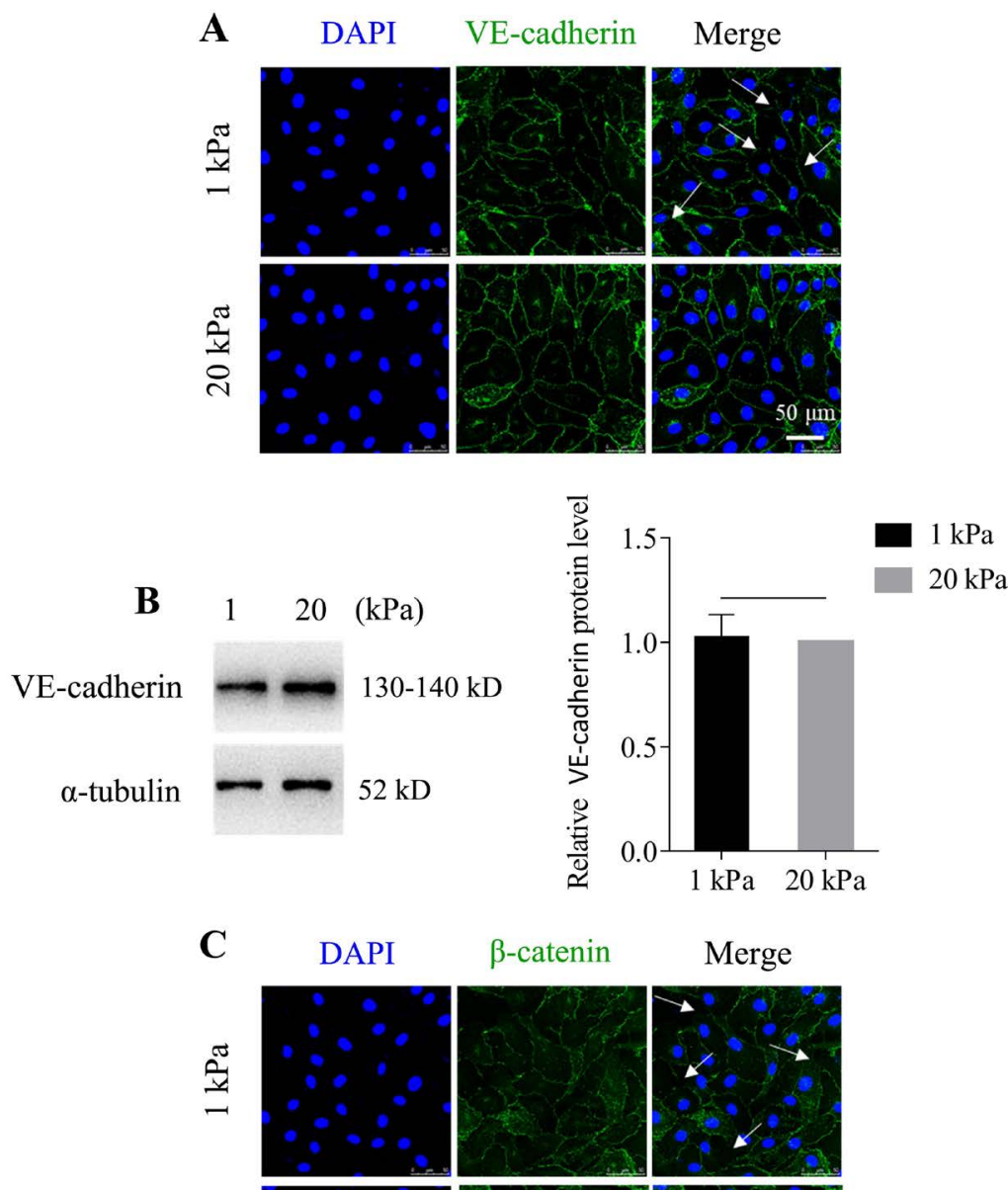

$\beta$-catenin

Merge
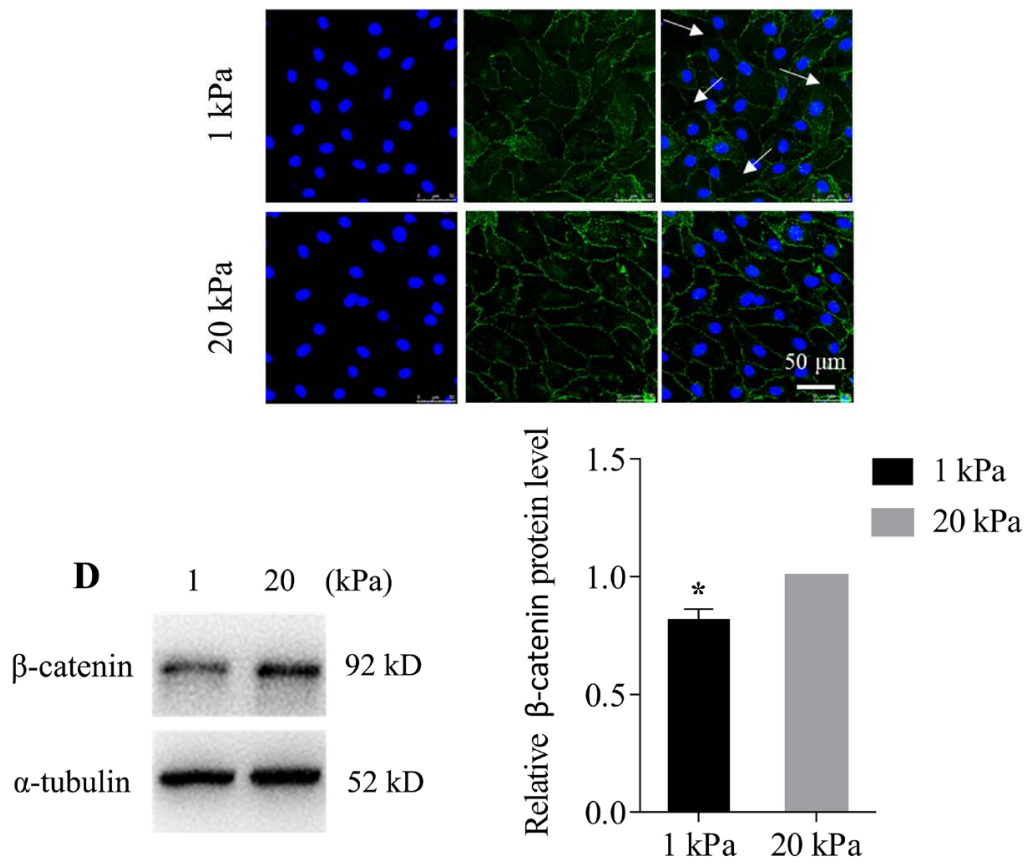

Figure 3. Soft substrate induced impaired AJs between ECs. ECs were cultured to reach a monolayer on PA gels. (A) Immunostaining of VE-cadherin (green). blue, DAPI staining of the nucleus. (B) The protein expression level of VE-cadherin in ECs. $n=3,{ }^{*} \mathrm{p}<0.05$. (Quantitation using $20 \mathrm{kPa}$ as the control.) (C) Immunostaining of $\beta$-catenin (green). blue, DAPI staining of the nucleus. (D) The protein expression level of $\beta$-catenin in ECs. $\mathrm{n}=3,{ }^{\star} \mathrm{p}<0.05$. (Quantitation using $20 \mathrm{kPa}$ as the control.) 
the protein levels of VE-cadherin in the ECs cultured on 1 and $20 \mathrm{kPa}$ PA hydrogels did not show significant difference (Figure 3(B)), the protein levels of $\beta$-catenin demonstrated a significant decrease in ECs on soft substrate (Figure 3(D)). In short, soft substrate could lead to disrupted AJs between ECs.

\section{Discussion}

The endothelium is critical for the normal function of the blood vessels. It forms a barrier to guarantee no circulating cells and soluble factors leaking into the tissues when the vessels deliver nutrients to tissues and regulates vasomotor tone sophisticatedly by synthesizing and secreting NO. Many vascular diseases, such as AS, are closely related to ECs dysfunction. AS starts with the abnormal accumulation of the various lipoproteins in the circulation, which may activate ECs to demonstrate inflammatory response by attracting circulating monocytes to adhere and extravasate through vascular wall [10]. If severe inflammation occurs after implanting a vascular scaffold or an artificial vessel replacement, it may possibly cause implantation failure. Therefore, proper strategies might be taken to adjust the characteristic of the material to avoid severe inflammation response and minimize implantation failure.

In this study, we found that soft substrate with stiffness around $1 \mathrm{kPa}$ could induce ECs inflammatory response. Compared with those cultured on substrate with normal stiffness of $20 \mathrm{kPa}$, ECs on the soft substrate express more ICAM1 and VCAM1, and have more monocyte adherence (Figure 1). Consistently, classic inflammation related transcription factor NF- $\kappa$ B was activated in the ECs on the soft substrate (Figure 2). Moreover, soft substrate leads to impaired AJs in EC monolayer, suggesting that the integrity of the blood vessel can be compromised in vivo (Figure 3 ).

The mechanism of how substrate stiffness affects EC inflammation is still under investigation. Mechanosensitive molecules like adhesion molecules, cytoskeletal components, various ion channels, G-protein-coupled receptors along with small GTPases and their effectors may be the mediators between the extracellular mechanical signal and the ECs function [26]. For example, the focal adhesion complex mediates the contact between cells and the extracellular matrix and has been reported to influence a variety of biological responses. Soft substrate may disrupt cell-cell junction by changing the activity of focal adhesion kinases, the assembly of structural proteins in the focal adhesion and the activity of integrins [27] [28] [29]. Cytoskeleton connects with focal adhesion and cell-cell junction. It has been reported that cytoskeleton mediated the clustering of VCAM1 and ICAM1 [30], so it may also be an important signaling transduction from the stiffness of the substrate to various ECs responses. In addition, ion channels located on the plasma membrane have been reported to react to the mechanical stimulation rapidly within microseconds, which was also involved in the intracellular signaling [31] [32], thus participating in many physiological activities of cells like inflammatory responses and AJs integrity. 
Here we found soft substrate induced EC inflammation and impaired AJs integrity. Our finding helps to further understand the mechanism of AS in the early stage and may provide some hints for the treatment of the disease.

\section{Conflicts of Interest}

The authors declare no conflicts of interest regarding the publication of this paper.

\section{References}

[1] Hoda, S.A. and Hoda, R.S. (2005) Robbins and Cotran Pathologic Basis of Disease. Anatomic Pathology, 12, 103.

[2] Ridker, P.M. (2019) Anticytokine Agents: Targeting Interleukin Signaling Pathways for the Treatment of Atherothrombosis. Circulation Research, 124, 437-450. https://doi.org/10.1161/CIRCRESAHA.118.313129

[3] VanderLaan, P.A., Reardon, C.A. and Getz, G.S. (2004) Site Specificity of Atherosclerosis: Site-Selective Responses to Atherosclerotic Modulators. Arteriosclerosis, Thrombosis, and Vascular Biology, 24, 12-22. https://doi.org/10.1161/01.ATV.0000105054.43931.f0

[4] Chiu, J. and Chien, S. (2011) Effects of Disturbed Flow on Vascular Endothelium: Pathophysiological Basis and Clinical Perspectives. Physiological Reviews, 91, 327-387. https://doi.org/10.1152/physrev.00047.2009

[5] Matsumoto, T., Abe, H., Ohashi, T., Kato, Y. and Sato, M. (2002) Local Elastic Modulus of Atherosclerotic Lesions of Rabbit Thoracic Aortas Measured by Pipette Aspiration Method. Physiological Measurement, 23, 635-648. https://doi.org/10.1088/0967-3334/23/4/304

[6] Chen, W., Tian, B., Liang, J., Yu, S., Zhou, Y. and Li, S. (2019) Matrix Stiffness Regulates the Interactions between Endothelial Cells and Monocytes. Biomaterials, 221, Article ID: 119362. https://doi.org/10.1016/j.biomaterials.2019.119362

[7] Tian, B., Ding, X., Song, Y., Chen, W., Liang, J., Yang, L., Fan, Y., Li, S. and Zhou, Y. (2019) Matrix Stiffness Regulates SMC Functions via TGF- $\beta$ Signaling Pathway. Biomaterials, 221, Article ID: 119407. https://doi.org/10.1016/j.biomaterials.2019.119407

[8] Moore, K.J. and Tabas, I. (2011) Macrophages in the Pathogenesis of Atherosclerosis. Cell, 145, 341-355. https://doi.org/10.1016/j.cell.2011.04.005

[9] Jonsson, A.L. and Bäckhed, F. (2017) Role of Gut Microbiota in Atherosclerosis. Nature Reviews Cardiology, 14, 79-87. https://doi.org/10.1038/nrcardio.2016.183

[10] Kobiyama, K. and Ley, K. (2018) Atherosclerosis. Circulation Research, 123, 1118-1120. https://doi.org/10.1161/CIRCRESAHA.118.313816

[11] Simionescu, M., Simionescu, N. and Palade, G.E. (1975) Segmental Differentiations of Cell Junctions in the Vascular Endothelium. The Microvasculature. Journal of Cell Biology, 67, 863-885. https://doi.org/10.1083/jcb.67.3.863

[12] Lampugnani, M.G., Corada, M., Caveda, L., Breviario, F., Ayalon, O., Geiger, B. and Dejana, E. (1995) The Molecular Organization of Endothelial Cell to Cell Junctions: Differential Association of Plakoglobin, Beta-Catenin, and Alpha-Catenin with Vascular Endothelial Cadherin (VE-Cadherin). Journal of Cell Biology, 129, 203-217. https://doi.org/10.1083/jcb.129.1.203

[13] Gong, H., Gao, X., Feng, S., Siddiqui, M.R., Garcia, A., Bonini, M.G., Komarova, Y., 
Vogel, S.M., Mehta, D. and Malik, A.B. (2014) Evidence of a Common Mechanism of Disassembly of Adherens Junctions through G $\alpha 13$ Targeting of VE-Cadherin. Journal of Experimental Medicine, 211, 579-591. https://doi.org/10.1084/jem.20131190

[14] Nottebaum, A.F., Cagna, G., Winderlich, M., Gamp, A.C., Linnepe, R., Polaschegg, C., Filippova, K., Lyck, R., Engelhardt, B., Kamenyeva, O., Bixel, M.G., Butz, S. and Vestweber, D. (2008) VE-PTP Maintains the Endothelial Barrier via Plakoglobin and Becomes Dissociated from VE-Cadherin by Leukocytes and by VEGF. Journal of Experimental Medicine, 205, 2929-2945. https://doi.org/10.1084/jem.20080406

[15] Schulte, D., Küppers, V., Dartsch, N., Broermann, A., Li, H., Zarbock, A., Kamenyeva, O., Kiefer, F., Khandoga, A., Massberg, S. and Vestweber, D. (2011) Stabilizing the VE-Cadherin-Catenin Complex Blocks Leukocyte Extravasation and Vascular Permeability. EMBO Journal, 30, 4157-4170.

https://doi.org/10.1038/emboj.2011.304

[16] Tse, J.R. and Engler, A.J. (2010) Preparation of Hydrogel Substrates with Tunable Mechanical Properties. In: Current Protocols in Cell Biology, John Wiley \& Sons, Hoboken, Chapter 10, Unit 10.16.

[17] Denisin, A.K. and Pruitt, B.L. (2016) Tuning the Range of Polyacrylamide Gel Stiffness for Mechanobiology Applications. ACS Applied Materials \& Interfaces, 8, 21893-21902. https://doi.org/10.1021/acsami.5b09344

[18] Tracqui, P., Broisat, A., Toczek, J., Mesnier, N., Ohayon, J. and Riou, L. (2011) Mapping Elasticity Moduli of Atherosclerotic Plaque in Situ via Atomic Force Microscopy. Journal of Structural Biology, 174, 115-123. https://doi.org/10.1016/j.jsb.2011.01.010

[19] Weber, C. and Noels, H. (2011) Atherosclerosis: Current Pathogenesis and Therapeutic Options. Nature Medicine, 17, 1410-1422. https://doi.org/10.1038/nm.2538

[20] Parry, G.C. and Mackman, N. (1994) A Set of Inducible Genes Expressed by Activated Human Monocytic and Endothelial Cells Contain Kappa B-Like Sites That Specifically Bind c-Rel-p65 Heterodimers. Journal of Biological Chemistry, 269, 20823-20825. https://doi.org/10.1016/S0021-9258(17)31895-1

[21] Stade, B.G., Messer, G., Riethmüller, G. and Johnson, J.P. (1990) Structural Characteristics of the 5' Region of the Human ICAM-1 Gene. Immunobiology, 182, 79-87. https://doi.org/10.1016/S0171-2985(11)80585-1

[22] Cybulsky, M.I., Allan-Motamed, M. and Collins, T. (1993) Structure of the Murine VCAM1 Gene. Genomics, 18, 387-391. https://doi.org/10.1006/geno.1993.1480

[23] Karin, M. (2008) The IkappaB Kinase-A Bridge between Inflammation and Cancer. Cell Research, 18, 334-342. https://doi.org/10.1038/cr.2008.30

[24] Daniel, A.E. and van Buul, J.D. (2013) Endothelial Junction Regulation: A Prerequisite for Leukocytes Crossing the Vessel Wall. Journal of Innate Immunity, 5, 324-335. https://doi.org/10.1159/000348828

[25] Lampugnani, M.G., Dejana, E. and Giampietro, C. (2018) Vascular Endothelial (VE)-Cadherin, Endothelial Adherens Junctions, and Vascular Disease. Cold Spring Harbor Perspectives in Biology, 10, a029322. https://doi.org/10.1101/cshperspect.a029322

[26] Douguet, D., Patel, A., Xu, A., Vanhoutte, P.M. and Honoré, E. (2019) Piezo Ion Channels in Cardiovascular Mechanobiology. Trends in Pharmacological Sciences, 40, 956-970. https://doi.org/10.1016/j.tips.2019.10.002

[27] Yang, X.N., Lu, Y.P., Liu, J.J., Huang, J.K., Liu, Y.P., Xiao, C.X., Jazag, A., Ren, J.L. and Guleng, B. (2014) Piezol Is as a Novel Trefoil Factor Family 1 Binding Protein 
That Promotes Gastric Cancer Cell Mobility In Vitro. Digestive Diseases and Sciences, 59, 1428-1435. https://doi.org/10.1007/s10620-014-3044-3

[28] Chen, X., et al. (2018) A Feedforward Mechanism Mediated by Mechanosensitive Ion Channel PIEZO1 and Tissue Mechanics Promotes Glioma Aggression. Neuron, 100, 799-815.e7. https://doi.org/10.1016/j.neuron.2018.09.046

[29] McHugh, B.J., Murdoch, A., Haslett, C. and Sethi, T. (2012) Loss of the Integrin-Activating Transmembrane Protein Fam38A (Piezo1) Promotes a Switch to a Reduced Integrin-Dependent Mode of Cell Migration. PLoS ONE, 7, e40346. https://doi.org/10.1371/journal.pone.0040346

[30] Scott, H.A., Quach, B., Yang, X., Ardekani, S., Cabrera, A.P., Wilson, R., Messaoudi-Powers, I. and Ghosh, K. (2016) Matrix Stiffness Exerts Biphasic Control over Monocyte-Endothelial Adhesion via Rho-Mediated ICAM-1 Clustering. Integrative Biology, 8, 869-878. https://doi.org/10.1039/C6IB00084C

[31] Delmas, P. and Coste, B. (2013) Mechano-Gated Ion Channels in Sensory Systems. Cell, 155, 278-284. https://doi.org/10.1016/j.cell.2013.09.026

[32] Murthy, S.E., Dubin, A.E. and Patapoutian, A. (2017) Piezos Thrive under Pressure: Mechanically Activated Ion Channels in Health and Disease. Nature Reviews Molecular Cell Biology, 18, 771-783. https://doi.org/10.1038/nrm.2017.92 